\title{
Circumcision among men who have sex with men in Scotland: limited potential for HIV prevention
}

\author{
Lisa M McDaid, ${ }^{1}$ Helen A Weiss, ${ }^{2}$ Graham $\mathrm{J} \mathrm{Hart}^{3}$
}

${ }^{1} \mathrm{MRC} / \mathrm{CSO}$ Social and Public Health Sciences Unit, Glasgow, UK

2MRC Tropical Epidemiology Group, London School of Hygiene and Tropical Medicine, London, UK

${ }^{3}$ Centre for Sexual Health and HIV Research, Research Department of Infection and Population Health, University College London, London, UK

\section{Correspondence to} Dr Lisa M McDaid, MRC/CSO

Social and Public Health Sciences Unit, 4 Lilybank Gardens, Glasgow G12 8RZ, UK; I.mcdaid@sphsu.mrc.ac.uk

Accepted 23 April 2010 Published Online First 30 June 2010

\section{(2) UNLOCK:}

This paper is freely available online under the BMJ Journals unlocked scheme, see http://sti. bmi.com/site/about/unlocked. xhtml

\section{ABSTRACT}

Objective Male circumcision has been shown to reduce the risk of HIV acquisition among heterosexual men but the impact among men who have sex with men (MSM) is not known. In this paper, we explore the feasibility of research into circumcision for HIV prevention among MSM in Scotland.

Methods Anonymous, self-complete questionnaires and Orasure oral fluid collection kits were distributed to men visiting the commercial gay scenes in Glasgow and Edinburgh.

Results 1508 men completed questionnaires $(70.5 \%$ response rate) and 1277 provided oral fluid samples (59.7\% response rate). Overall, 1405 men were eligible for inclusion in the analyses. 16.6\% reported having been circumcised. HIV prevalence was similar among circumcised and uncircumcised men $(4.2 \%$ and $4.6 \%$, respectively). Although biologically, circumcision is most likely to protect against HIV for men practising unprotected insertive anal intercourse (UIAI), only 7.8\% (91/1172) of uncircumcised men reported exclusive UIAI in the past 12 months. Relatively few men reported being willing to participate in a research study on circumcision and HIV prevention (13.9\%), and only $11.3 \%$ of uncircumcised men did so.

Conclusion The lack of association between circumcision and HIV status, low levels of exclusive UIAI, and low levels of willingness to take part in circumcision research studies suggest circumcision is unlikely to be a feasible HIV prevention strategy for MSM in the UK. Behaviour change should continue to be the focus of HIV prevention in this population.

Three randomised controlled trials have shown that male circumcision reduces the risk of HIV acquisition among heterosexual men by approximately $60 \%,{ }^{1}$ and male circumcision is now recommended as an additional HIV prevention strategy for this population. ${ }^{2}$ However, the evidence for such an association among men who have sex with men (MSM) is weak and inconsistent. ${ }^{3-5}$ Biologically, circumcision may provide partial protection against HIV acquisition among MSM practising unprotected insertive anal intercourse (UIAI), in a similar way as it protects against vaginal-penile transmission. Possible mechanisms include the fact that the thin inner surface of the foreskin is susceptible to microtears and abrasions, and contains a high density of superficial Langerhan's cells; and the preputial space provides an environment thought to favour pathogen survival and replication. ${ }^{4-9}$

In the UK, MSM are the group most at risk of acquiring HIV, with an estimated prevalence of $5 \%,{ }^{10}$ and new HIV prevention strategies are needed. Circumcision is not common in the UK, ${ }^{11}$ and attitudes towards circumcision are largely unknown. One recent survey of London MSM found only one in 10 uncircumcised men were willing to participate in future research on circumcision for HIV prevention. ${ }^{12}$ The aim of our study was to describe sexual practices by circumcision status, and to explore the feasibility of conducting research on male circumcision for HIV prevention among MSM in Scotland.

\section{METHODS}

Circumcision questions were included in the 2008 MRC Gay Men's Survey, which collected anonymous, self-complete questionnaires and (Orasure) oral fluid specimens (screened for anti-HIV using an enzyme immunoassay; positives re-screened, and repeat reactives confirmed using western blot). Time and location sampling was used to recruit representative samples in the commercial gay scenes of Glasgow and Edinburgh. ${ }^{13}$ Data were analysed using SPSS 15.0. Logistic regression was used to estimate OR and $95 \%$ CI. OR were adjusted for age and nationality, which were significantly associated with circumcision status. Ethical approval was granted by the University of Glasgow, Faculty of Medicine Ethics Committee.

\section{RESULTS}

Two thousand one hundred and thirty-eight men were approached, 1508 completed questionnaires (70.5\% response rate) and 1277 provided oral fluid samples $(59.7 \%$ response rate). In multivariate analysis comparing men who did and did not provide samples, only age was significant (reduced odds among men aged over 26 years, adjusted OR $0.63,95 \%$ CI 0.43 to 0.92 ). Fifty-four men who reported they were heterosexual and had no sexual contact with men in the previous 12 months and 49 men who did not provide their circumcision status are excluded ( $\mathrm{N}=1405)$.

Two hundred and thirty-three men (16.6\%) reported having been circumcised; $32.3 \%$ had been circumcised before they were 1 year old and, overall, $73.1 \%$ by the age of 18 years. Compared with the 16-25 years age group, men aged $36-45$ and over 46 years were more likely to be circumcised $(21.4 \%$ and $18.2 \%$, respectively, compared with $11.8 \%, p=0.007)$. Nationals from non-European countries (mainly America, Canada, Australia and New Zealand) were more likely to be circumcised than Scottish men $(50.0 \%$ and $13.1 \%$, respectively, $\mathrm{p}<0.001$ ).

HIV prevalence was comparable among uncircumcised and circumcised men $(4.6 \%$ and $4.2 \%$, respectively; table 1). Similarly, there was little difference in prevalence of other, self-reported, sexually transmitted infection (STI) in the past 
Table 1 HIV status, sexual behaviour and willingness to take part in a research study on circumcision and HIV prevention: comparing circumcised and uncircumcised men

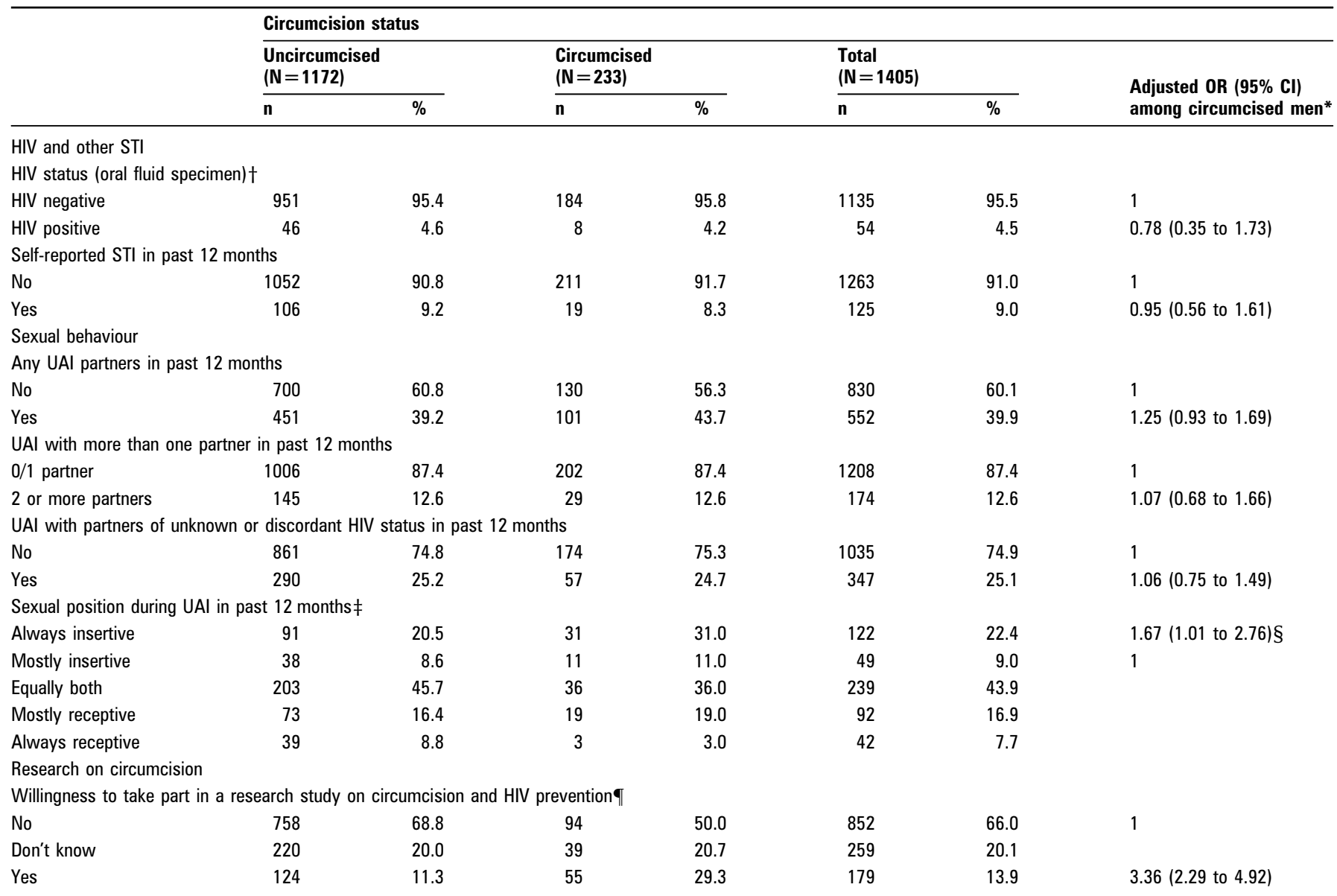

*Adjusted for age and nationality.

†Among men who provided oral fluid specimens $(N=1189)$.

$\ddagger$ Among men reporting any unprotected anal intercourse (UAI) ( $N=552$ ).

§Adjusted OR for men reporting always being the insertive UAI partner in the past 12 months; for men reporting always or mostly being the insertive UAI partner in the past 12 months adjusted OR $1.82,95 \%$ Cl 1.14 to 2.92 .

ๆThe willingness question used was 'We are looking for new ways to prevent HIV. Should the following research studies take place, which would you be willing to take part in?', with participants asked to select 'yes', 'no' or 'don't know' for circumcision (and also for behaviour change, rectal microbicides and HIV vaccines).

STI, sexually transmitted infection.

12 months (although it should be noted that STI transmission may also occur by means other than anal intercourse).

Overall, $39.9 \%$ of men reported any unprotected anal intercourse in the past 12 months and this was similar by circumcision status (table 1 ). Of these, $22.4 \%$ reported exclusive UIAI, and circumcised men were more likely to report this than uncircumcised men (table 1). Among men who reported always being the insertive partner, none of the circumcised men tested HIV positive (0/27), compared with $2.5 \%(2 / 79)$ of uncircumcised men.

Few men $(13.9 \%)$ reported being willing to take part in an HIV prevention research study on circumcision, while $20.1 \%$ reported that they did not know if they would be willing to do so (table 1). Only $11.3 \%$ of uncircumcised men reported being willing to take part.

\section{DISCUSSION}

This is the first study to assess the association between circumcision and HIV among MSM in Scotland, although some study limitations should be noted. The data are cross-sectional and causality cannot be inferred. The oral fluid sample response rate was relatively low and may not be representative. However, the overall survey response rate was higher (71\%), and only age differed between the men who did and did not provide oral fluid specimens; suggesting that the men who provided these were representative of the larger venue-based sample. Finally, circumcision status was self-reported, although this has been shown to be a valid measure. ${ }^{14}$

We found no evidence of an association between circumcision and HIV or other self-reported STI among MSM in Scotland, similar to findings elsewhere. ${ }^{3-5}$ Among men reporting unprotected anal intercourse, less than one-quarter exclusively favoured the insertive role, which might plausibly provide partial protection against HIV infection. In contrast to a recent US study, ${ }^{15}$ this behaviour was more common among circumcised than uncircumcised men; and none of the circumcised men who were exclusively the insertive partner tested HIV positive. Although findings are inconsistent across studies, a possibly protective effect among this group has been reported, ${ }^{4}$ and merits further investigation.

There are few studies of the willingness of uncircumcised MSM to be circumcised. ${ }^{4}$ One, from the USA, found that $53 \%$ were willing to be circumcised. ${ }^{16}$ However, our findings are similar to those of the London study, in which only one in 10 uncircumcised men were willing to participate in circumcision research. ${ }^{12}$ Together with the low levels of exclusive UIAI in these populations, these findings suggest that a randomised 


\section{Key messages}

There was no evidence of an association between circumcision and HIV or other self-reported STI among MSM in Scotland.

- Among men reporting unprotected anal intercourse, less than one-quarter exclusively favoured the insertive role.

- With only $11 \%$ of uncircumcised men reporting willingness to take part in a HIV prevention research study on male circumcision, a randomised controlled trial of male circumcision for HIV prevention is unlikely to be feasible in this population.

controlled trial of male circumcision for HIV prevention is unlikely to be feasible in the UK. Although circumcision may be partly protective against HIV infection for those men who are exclusively insertive partners, our results suggest that comprehensive HIV prevention strategies, including the promotion of consistent condom use, should continue to be the focus of prevention efforts among MSM.

Acknowledgements The authors would like to thank the survey staff and fieldworkers in each city, the venue managers, their staff and the men who agreed to participate in the survey.

Funding The UK Medical Research Council funds LMMcD and the Gay Men's Survey as part of the Sexual and Reproductive Health Programme (WBS U.1300.00.005) at the Social and Public Health Sciences Unit, and funds HAW as part of the MRC Tropical Epidemiology Group.

Competing interests None.

Ethics approval This study was conducted with the approval of the University of Glasgow Faculty of Medicine Ethics Committee.

Contributors $L M M C D$, HAW and GJH devised the paper. LMMcD conducted the analyses and wrote the first draft. All authors contributed to subsequent drafts and approved the final version of the manuscript.

Provenance and peer review Not commissioned; externally peer reviewed.

\section{REFERENCES}

1. Weiss HA, Halperin D, Bailey RC, et al. Male circumcision for HIV prevention: from evidence to action? AIDS 2008;22:567-74.

2. WHO/UNAIDS. New data on male circumcision and HIV prevention: policy and programme implications. Geneva: WHO, 2007. http://www.who.int/hiv/pub/ malecircumcision/research implications/en/index.html (accessed 21 Dec 2009).

3. Millett GA, Flores SA, Marks G, et al. Circumcision status and risk of HIV and sexually transmitted infections among men who have sex with men. JAMA 2008;300:1674-84.

4. Templeton DJ, Millett GA, Grulich AE. Male circumcision to reduce the risk of HIV and sexually transmitted infections among men who have sex with men. Curr Opin Infect Dis 2010;23:45-52

5. Gust DA, Wiegand RE, Kretsinger $\mathrm{K}$, et al. Circumcision status and HIV infection among MSM: reanalysis of a phase III HIV vaccine clinical trial. AIDS 2010;24:1135-43.

6. Donoval BA, Landay AL, Moses S, et al. HIV-1 target cells in foreskins of African men with varying histories of sexually transmitted infections. Am J Clin Pathol 2006;125:386-91.

7. McCoombe SG, Short RV. Potential HIV-1 target cells in the human penis. AIDS 2006;20:1491-5.

8. Patterson BK, Landay A, Siegel JN, et al. Susceptibility to human immunodeficiency virus-1 infection of human foreskin and cervical tissue grown in explant culture. Am J Pathol 2002;161:867-73.

9. Szabo R, Short RV. How does male circumcision protect against HIV infection? BMJ 2000;320:1592-4.

10. Health Protection Agency. Sexually transmitted infections and men who have sex with men in the UK: 2008 report. London: Health Protection Agency, 2008.

11. Dave SS, Johnson AM, Fenton KA, et al. Male circumcision in Britain: findings from a national probability sample survey. Sex Transm Infect 2003; 79:499-500.

12. Thornton A, Lattimore $S$, Delpech $V$, et al. Circumcision among men who have sex with men in London (Abstract No P4.62). 18th Biennial Meeting of the International Society for STD Research. 28 June-1 July 2009; London, UK. International Society for STD Research/British Association for Sexual Health and HIV.

13. Williamson LM, Hart GJ. HIV prevalence and undiagnosed infection among a community sample of gay men in Scotland. J Acquir Immune Defic Syndr 2007:45:224-30

14. Templeton DJ, Mao L, Prestage GP, et al. Self-report is a valid measure of circumcision status in homosexual men. Sex Transm Infect 2008;84:187-8.

15. Jameson DR, Celum CL, Manhart L, et al. The association between lack of circumcision and HIV, HSV-2, and other sexually transmitted infections among men who have sex with men. Sex Transm Dis 2010;37:147-52.

16. Begley EB, Jafa $\mathrm{K}$, Voetsch AC, et al. Willingness of men who have sex with men (MSM) in the United States to be circumcised as adults to reduce the risk of HIV infection. PLoS One 2008:3:e2731. 